\title{
Prevalence of Meningioma in South Indian Population Diagnosed through Magnetic Resonance Spectroscopy - A Retrospective Study
}

\author{
Smitha $\mathbf{H}^{1}$, Sreekanth K Sivaraman ${ }^{2, *}$ \\ 1Department of Physiology, Sree Gokulam Medical College and Research Foundation, Venjaramoodu, Trivandrum, Kerala, INDIA. \\ ${ }^{2}$ Department of Molecular Biology, Sree Gokulam Medical College and Research Foundation, Venjaramoodu, Trivandrum, Kerala, INDIA.
}

\begin{abstract}
Introduction: Meningioma originates in cerebral dura mater and can grow at any site, especially at the skull vault and the skull base. Meningioma accounts for $37 \%$ of all central nervous system tumor. MRS (Magnetic Resonance Spectroscopy) provides additional structural and metabolic information that helps to diagnose meningioma. Objectives: The aim of the study was to investigate the prevalence of brain tumor meningioma in patients with characteristic risk factors like headache, vomiting, dizziness etc to assess the diagnostic significance. Materials and Methods: This is a retrospective study and was conducted in the Radiology Department and Biophysics Departments of one of the tertiary health care centres in South Kerala. This study was conducted on 81 subjects with brain lesion, including both males and females, who underwent MRS. From MRS findings, the prevalence of Meningioma was detected. Patient's demographics were also taken for the analysis and interpretation. Results: From this study it was understood that out of the 81 cases, 40 were of Meningioma. In this 40 patients, 22 were females (55\%) and 18 were males (45\%). Among these 40 cases, the frequency of brain lesions was higher in old aged group (60\%) when compared to younger $(0 \%)$ and middle age groups (40\%). It was found that out of the 40 patients, $42 \%$ had headache, $28 \%$ had vomiting, $23 \%$ had Type- 2 diabetes mellitus and $7 \%$ had dizziness. Conclusion: From this study, it is confirmed that among the brain lesions, the incidence of Meningioma is higher and was prominent among old aged group and the incidence is reported to be higher in females. This indicates the need of special attention for these patients for early diagnosis and timely intervention.
\end{abstract}

Key words: Brain lesions, Dizziness, Diabetes Mellitus, Gender, Meningioma, Vomiting.
Article Information

Received: 01-09-2021

Revised : 13-10-2021

Accepted: 18-12-2021

Correspondence
Dr. Sreekanth K Sivaraman
Professor of Biochemistry and HOD,
Department of Molecular Biology,
Foundation, Venjaramoodu,
Sree Gokulam Medical College and Research
Trivandrum-695607, Kerala, INDIA.
E-mail: drsreekanthtrivandrum@gmail.com

\section{INTRODUCTION}

Prevalence and incidence of brain lesions leading to meningioma are increasing in recent years and the underlying diseases associated with lesions are also

\begin{tabular}{|l|c|}
\hline \multicolumn{2}{|c|}{ Access this article online } \\
\hline $\begin{array}{l}\text { Website: } \\
\text { www.jcramonline.com }\end{array}$ & Quick Response code \\
\hline Dol: & \\
10.5530/jcram.1.2.15 & \\
& \\
\hline
\end{tabular}

drastically increasing. Associated symptoms commonly observed are headache, pain in the forehead and neck, memory loss, vomiting, seizures and confusion. However an early diagnosis may help to lead a quality life with proper treatment and medication.

Among the intracranial tumors, meningiomas are seen commonly. It was found that the incidence of meningioma were eight cases per 100,000 persons per year and majority of them are affected by adult females than adult males in US. ${ }^{1}$ Meningioma's originate in cerebral dura mater and can grow at any site, especially at the skull vault and the skull base. They are generally benign tumors and mostly asymptomatic. Meningiomas 
are the most frequent central nervous system neoplasm, it accounts for $37 \%$ of all central nervous system tumors. ${ }^{2}$ By the Finnish Cancer Registry (FCR), it was reported that the national incidence rates of meningiomas are 1.6 per 100000 for men and 5.5 for women in 2001. In the Nordic countries the corresponding rates were 1.9 and 4.5 per 100000 person respectively. The incidence rates in the United States, by the Central Brain Tumor Registry of the United States were 1.8 for men and 4.2 per 100000 for women in $2006 .^{3}$

In early stage, the symptom is determined as mild headaches, when the tumor progress, a large number of complications and poor functional outcomes will appear. ${ }^{4}$ The prevalence of previous history varies depending on location, type of tumour and age of the patient. ${ }^{2}$ After head injury at any age, vomiting is another common symptom..$^{5}$ In addition to this, a risk factor of brain injury is high and low blood pressure. ${ }^{6}$ The common clinical symptoms of meningiomas may include chronic headaches, dizziness, visual loss, and seizures.

Different MRI modalities have been used for meningioma differentiation from other intracranial brain tumor like Diffusion-weighted MRI (DWI), perfusionweighted MRI (PWI) and proton magnetic resonance spectroscopy ( $\left.{ }^{1} \mathrm{H}-\mathrm{MRS}\right) .{ }^{8}$ MRS provides additional structural and metabolic information. Proton magnetic resonance spectroscopy ( $\left.{ }^{1} \mathrm{H}-\mathrm{MRS}\right)$, is a non-invasive, quantitative technique for estimating metabolite / inside the / tumor. The metabolites include $\mathrm{N}$-acetylaspartate (NAA), creatine (Cr), and choline (Cho). ${ }^{9}$ The current study was undertaken to investigate the prevalence of brain tumor meningioma in patients with characteristic risk factors including headache, vomiting, dizziness etc to assess the diagnostic significance.

\section{MATERIALS AND METHODS}

This is a retrospective study and was conducted in the Radiology and Biophysics Departments of one of the major tertiary health care centres in South Kerala. The patients suspected of brain tumor lesions and underwent MRS for the diagnoses were included in the study during the period of January 2018 to December 2018. The study was approved by the Institutional Ethics Committee. Only patients with proven MRS diagnosis for brain lesions were included as study subjects. The brain tumor lesions patients suspecting meningioma, patient's demographic characters including age, gender and history were also taken for the analysis and interpretation. Both males and females were included in the study. Patients with implants, aneurysm clips, pacemakers, heart valves and those who are not willing to participate in the study and patients with age below 20 years were excluded from this study.

The patients were categorised according to the age as patients with $20-40$ years, patients with $40-60$ years and patients with $>60$ years. There were 81 brain lesion cases with both males and females, who underwent MRS for the diagnosis of meningioma and from this, 40 patients suspecting meningioma were included for further analysis. Patient's demographics including age, gender and history were also taken for the analysis and interpretation. MRI scans of three different planesAxial, Coronal and Sagittal were performed by using MRI scans of $1.5 \mathrm{~T}$ with sequences like FLAIR T2 weighted imaging and diffusion-weighted imaging (DWI) to determine the pathological conditions of cerebral tissue. For analysing the spectra, Spectroscopy-2D Brain software was used. MRS made sue of radiofrequency $(\mathrm{RF})$ coils and a software package. The MRS acquisition was started with anatomic images, which were used to select a volume of interest (VOI). For the spectrum acquisition, different spatial localization techniques (Single-voxel and Multi-voxel imaging) was used using both long and short echo times (TE). Single-voxel (SV) technique records spectra from one region of the brain at a time. Multi-voxel technique simultaneously recorded spectra from multiple regions.

\section{RESULTS}

Figure 1 shows the MRS spectrum of Meningioma and Table 1 gives the average values of observed metabolites analysed through Magnetic resonance Spectroscopy. It was found that the among the 40 meningioma tumors, the average values of observed metabolites like $\mathrm{N}$

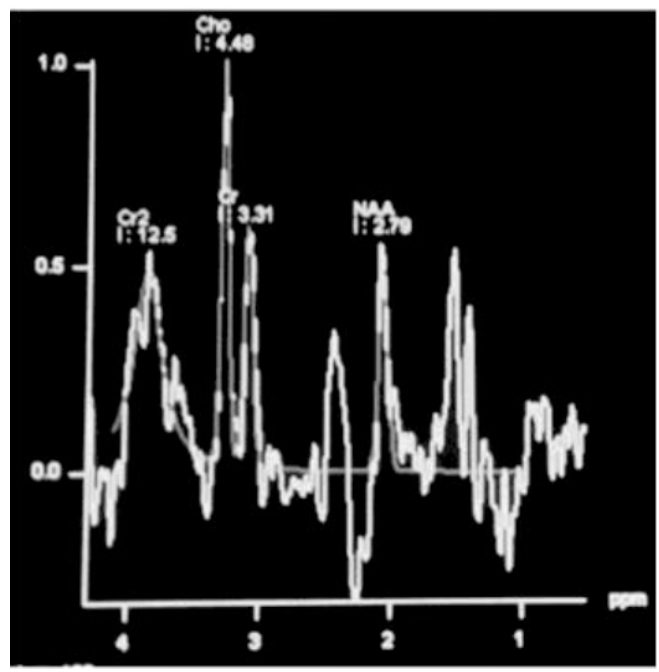

Figure 1: MRS spectrum of Meningioma. 
Table 1: Average values of Observed Metabolites of Meningioma analysed through Magnetic Resonance Spectroscopy.

\begin{tabular}{cccccc}
\hline \multirow{2}{*}{ Brain } & \multicolumn{2}{c}{ Observed Metabolites } & & \\
\cline { 2 - 7 } Tumor & N Acetyl Aspartate & Creatine $(\mathbf{C r})$ & Choline(Cho) & Alanine & Lipid \\
\hline & NAA 2.02 & $($ Cr)3.0 & 3.22 & 1.3 & $1.3-1.5$ \\
Meningioma & 1.86 & 2.44 & 3.68 & 1.35 & 1.43 \\
\hline
\end{tabular}

Acetyl Aspartate (NAA) (1.86 ppm) were decreased from normal value $(2.02 \mathrm{ppm})$. Similarly the Creatine (Cr) $(2.44 \mathrm{ppm})$ were also determined as decreased from normal value (3.0 ppm). But the Choline (Cho) (3.68 ppm) were indicated as increased from normal value (3.22 ppm). In addition to that specific metabolites Alanine were also observed at $1.35 \mathrm{ppm}$ and lipid at 1.43 ppm.

Figure 2 shows the distribution of meningioma according to gender. From this study it was observed that out of 40 meningioma patients analysed, 22 patients $(55 \%)$ were females and $18(45 \%)$ were males, showing a higher incidence of meningioma in Females. Figure 3 represents the frequency of brain lesions with respect to age in these subjects. Among these 40 cases, age varied from 20 years to 60 years. It was revealed that the frequency of brain lesions was higher in old age group $(60 \%)$ when compared to younger age $(0 \%)$ and middle age groups $(40 \%)$. Patients with age $20-40$ years accounted for zero cases (0\%), 40-60years accounted for 16 cases $(40 \%)$ and $>60$ years accounted for 24 cases $(60 \%)$. Figure 4 gives the history of other inflammatory diseases and symptoms shown by these participants. It was found that out of 40 patients, $42 \%$ was suffering from headache, $28 \%$ from vomiting, $23 \%$ from Type- 2 diabetes mellitus and $7 \%$ had dizziness.

\section{DISCUSSION}

Recently clinician takes keen interest in the management of brain tumor lesions due to their high incidence. In this study, the data is analyzed retrospectively, regarding demographics, incidence and the symptoms associated in the meningioma tumor, presented in patients who attended in a major tertiary health care centre in South Kerala over a period of 12 months. This information may help to predict the incidence of different types of meningioma brain tumor lesions and need for specific diagnosis to confirm the lesions as early as possible to get better treatment.

Present study shows that the MRS spectrum of meningioma (Figure 1) and Table 1 gives values of the peak NAA (1.86 ppm), which were significantly reduced. Similarly the concentration of Cho (3.68

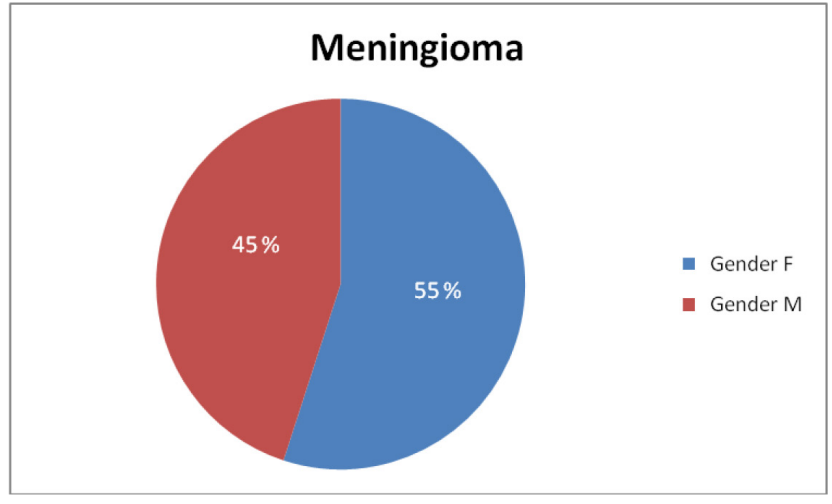

Figure 2: Distribution of Meningioma in patients according to gender.

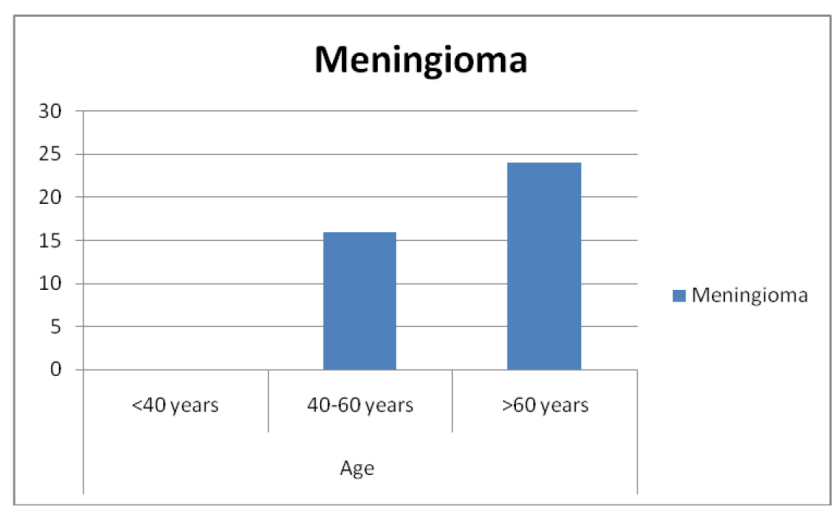

Figure 3: Incidence of Meningioma among the subjects according to age.

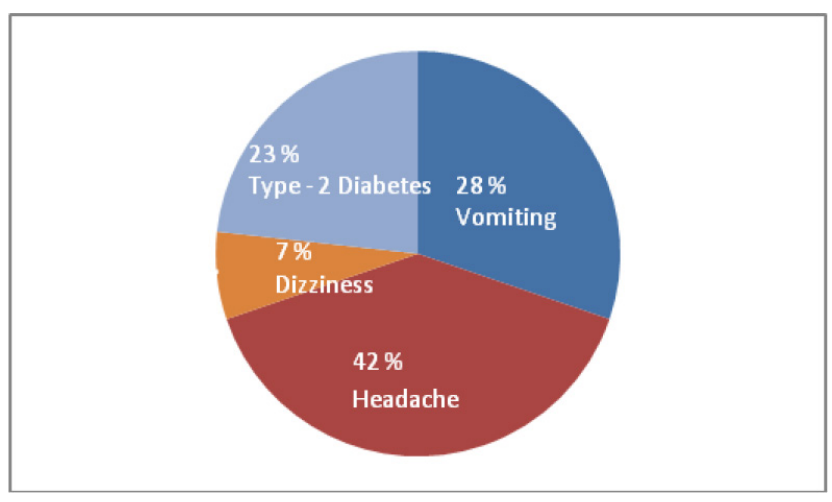

Figure 4: History of other inflammatory Diseases and Other Symptoms. 
ppm) were high and $\mathrm{Cr}$ (2.44 ppm) were low. Lipids (1.43 ppm) and alanine (1.35 ppm) were also present in higher concentrations in this study. Previous studies also determined that there is a presence of Alanine in meningioma, diagnosed through MRS. ${ }^{10}$

In this study it was observed that the old age group $(>60$ years) is more affected with meningioma brain tumor when compared to younger age and middle aged group. Previous studies also indicated that with increasing age, the incidence of meningioma also increases. It was found that the incidence rate of meningioma in the age of $\geq 65$ years is $12.8 / 100,000$ than $45-54$ year old $(4.9 / 100,000)$ and $55-64$ year old $(7.9 / 100,000) .{ }^{11}$ In another studies also it was confirmed that the older age groups are affected with meningioma more in number, as the incidence rate is higher in old age group than younger age group. ${ }^{12}$

Present study reveals that the brain tumor meningioma were found to be more in females compared to males. In one of the studies it was reported that the incidence of meningioma's are higher in women due to the hormonal difference. ${ }^{13}$ Earlier studies also indicated that the females are more prone to meningioma than males. ${ }^{14}$ In the current study it was found that the brain tumor, meningioma patients, were diagnosed with type -2 diabetes mellitus (DM). In one of the study, it was confirmed that the prevalence of diabetes was significantly higher in patients with meningioma compared to normal population. ${ }^{15}$ Present study determined that the meningioma patients had the symptom of headache. Earlier studies also showed that in the concurrence of headache was found in patients with meningioma. ${ }^{16}$ In another study also it was mentioned that headache is one of the symptom which is associated with meningioma. ${ }^{17-19}$ In this study it was observed that the meningioma was associated with vomiting. In one of the study it was indicated that one of the symptoms of meningioma is vomiting. ${ }^{17} \mathrm{In}$ another study it was also realised that the occurrence of vomiting is present in the meningioma patients. ${ }^{20}$ In the present study it was observed that the occurrence of dizziness is another symptom of meningioma. In the earlier studies also it was reported that dizziness is one of the associated symptoms in meningioma patients. ${ }^{21}$ It was described in another study also that dizziness is one of the symptoms in patients suffering from meningioma. ${ }^{22}$

\section{CONCLUSION}

The incidence of Brain lesions are increasing and the best diagnostic tool for the detection and identification of lesions is Magnetic Resonance Spectroscopy (MRS). Among the brain lesions the incidence of meningioma is higher and it was prominent among the old age group of South Indian population. It is found that the prevalence of meningioma is higher in females as compared to males. These findings may help the clinicians for an early diagnosis and management of brain lesions diagnosed through Magnetic Resonance Spectroscopy. Management of persistent symptoms and other clinical condition is based on assessment of risk and understanding of potential complications related to the diseases. This may lead to better categorization of symptom constructs and prospective trials for the management of the symptoms in patients with meningioma.

\section{ACKNOWLEDGEMENT}

The authors sincerely thank the Director and the Management of Sree Gokulam Medical College and Research Foundation for providing the facility to carry out the research work. They also like to acknowledge the study participants.

\section{CONFLICT OF INTEREST}

The authors declare that there is no conflict of interest.

\section{REFERENCES}

1. BerndHolleczek Daniel. Zampella, Steffi Urbschat, Felix Sahm, Andreasvon Deimling, Joachim Oertel, RalfKetter, Incidence, mortality and outcome of meningiomas: A population-based study from Germany. Cancer Epidemiol. 2019;62:1-8.

2. Kirby S, Purdy RA. Headache and brain tumors. Curr Neurol Neurosci Rep. 2007;7(2):110-6. doi: 10.1007/s11910-007-0005-7.

3. Larjavaara $S$, Haapasalo $H$, Sankila $R$, Helén $P$, Auvinen $A$. Is the incidence of meningiomas underestimated? A regional survey. $\mathrm{Br} \mathrm{J}$ Cancer. 2008;99(1):182-4. doi: 10.1038/sj.bjc.6604438, PMID 18577989.

4. Lin Dong-Dong, Lin Jia-Liang, Deng Xiang-Yang, Li Wei, Li Dan-Dong, Yin B, et al. Trends in intracranial meningioma incidence in the United States, 20042015. Cancer Med. 2019;8(14):6458-67. doi: 10.1002/cam4.2516, PMID 31475482.

5. Borland Meredith L, Dalziel Stuart R, Phillips Natalie, Dalton Sarah, Lyttle Mark D, Bressan Silvia, et al. Paediatric Research in Emergency Department International Collaborative group. Vomiting with head trauma and risk of traumatic brain injury. Pediatrics. 2018;141(4):1-12. doi: 10.1542/peds.20173123, PMID 29599113.

6. Jain Vivek, Choudhary Jitendra, Pandit Rahul. Blood pressure target in acute brain injury. Indian J Crit Care Med. 2019;23(Suppl 2);Suppl 2:S136-9. doi: 10.5005/jp-journals-10071-23191, PMID 31485122.

7. Bhala Sonia, Stewart Douglas R, Kennerley Victoria, Petkov Valentina I, Rosenberg Philip S, Best Ana F. Incidence of benign meningiomas in the United States: Current and future trends. JNCl Cancer Spectr. 2021;5(3):pkab035. doi: 10.1093/jncics/pkab035, PMID 34250440.

8. Kousi Evanthia, Tsougos loannis, Fountas Konstantinos, Theodorou Kiriaki, Tsolaki Evaggelia, Fezoulidis loannis, et al. Distinct peak at 3.8 ppm observed by $3 T$ MR spectroscopy in meningiomas, while nearly absent in highgrade gliomas and cerebral metastases. Mol Med Rep. 2012;5(4):1011-8. doi: 10.3892/mmr.2012.773, PMID 22293950. 
9. Lin MC, Li CZ, Hsieh CC, Hong KT, Lin BJ, Lin C, et al. Preoperative grading of intracranial meningioma by magnetic resonance spectroscopy (1H-MRS). PLOS ONE. 2018;13(11):1-8. doi: 10.1371/journal.pone.0207612.

10. Horská Alena, Barker Peter B. Imaging of brain tumors: MR spectroscopy and metabolic imaging. Neuroimaging Clin N Am. 2010 Aug;20(3):293-310. doi: 10.1016/j.nic.2010.04.003, PMID 20708548.

11. Kaul David, Budach Volker, Graaf Lukas, Gollrad Johannes, Badakhshi Harun. Outcome of elderly patients with meningioma after image-guided stereotactic radiotherapy: A study of 100 cases. Biomed Res Int. 2015;2015:868401. doi: 10.1155/2015/868401, PMID 26101778.

12. Niiro M, Yatsushiro K, Nakamura K, Kawahara Y, Kuratsu J. Natural history of elderly patients with asymptomatic meningiomas. J Neurol Neurosurg Psychiatry. 2000;68(1):25-8. doi: 10.1136/jnnp.68.1.25, PMID 10601396.

13. Maiuri Francesco, Giuseppe Mariniello Teresa Somma, Elia Guadagno, Sergio Corvino, Serena Pagano, Valentina Orlando and Marialaura Del Basso De Caro. Meningiomas in Premenopausal Women: Role of the Hormone Related Conditions, Front. Oncol. 2020;10:1-8.

14. Sun Tao, Plutynski Anya, Ward Stacey, Rubin Joshua B. An integrative view on sex differences in brain tumors. Cell Mol Life Sci. 2015;72(17):3323-42. doi: 10.1007/s00018-015-1930-2, PMID 25985759.
15. Salehpoor Firooz, Pezeshki Arastoo, Haghir Amirhossein, Kazempoor Aidin, Mirzaei Farhad, Fekri Sanaz, et al. Meningioma and it's relation with Diabetes. Int J Curr Res Acad Rev. 2014;2(8):36-40.

16. Talacchi Andrea, Lombardo Cristina, Bricolo Albino. Vascular headache due to intracranial meningioma: A curable form of headache. Lancet. 1997;350(9083):1004-5. doi: 10.1016/S0140-6736(05)64070-6, PMID 9329522.

17. Bharti N, Kasyap.L, Mohan V K, Anesthetic management of a parturient with cerebellopontine angle meningioma. Case Report. 2002;11(3):219-21.

18. Falavigna A, Santos JA, Chimelli L, Ferraz FA, Bonatelli Ad Ade P. Anaplastic meningioma: Case report. Arq Neuropsiquiatr. 2001;59(4):939-43. doi: 10.1590/s0004-282x2001000600019. PMID 11733842.

19. Perkins Allen, Liu Gerald. Primary brain tumors in adults: Diagnosis and treatment. Am Fam Physician. 2016;93(3):211-7. PMID 26926614.

20. Goyal R, Gupta P. Clinicopathological study of meningioma from rural set up of central India: A 5 year experience. Indian J Pathol Oncol. 2019;6(4):539-42. doi: 10.18231/j.ijpo.2019.105.

21. Millet Arnaud, Morandi Xavier, Hamidou Mohamed, Jego Patrick. Intracranial meningioma mimicking a carcinoid tumor. Am J Med. 2008;121(5):e1-2. doi: 10.1016/j.amjmed.2008.01.027, PMID 18456011.

22. Pham Jason T, Kim Ronald C, Nguyen Anna, Bota Daniela, Kong Xiao Tang, Vadera Sumeet, et al. Intracranial meningioma with carcinoma tumor -totumor metastatis: Two case reports. CNS Oncol. 2017;7(2):1-8.

Cite this Article : Smitha H, Sivaraman SK. Prevalence of Meningioma in South Indian Population Diagnosed through Magnetic Resonance Spectroscopy - A Retrospective Study. J. Clin. Res. Applied Med. 2021;1(2):63-7. 